\title{
An Introduction to
}

\section{Programming}

with

Mathematica ${ }^{\circledR}$

\author{
Third Edition
}

Paul R. Wellin | Richard J. Gaylord | Samuel N. Kamin 


\section{Contents}

Preface $\cdot x i$

\section{An introduction to Mathematica}

\subsection{A brief overview of Matbematica 1}

Numerical computations · Symbolic computations - Graphics - Working with data .

Programming $\cdot$ Symbolic and interactive documents

1.2 Using Mathematica $\cdot 15$

Getting into and out of Mathematica . The syntax of inputs . Alternate input syntax . The front end and the kernel $\cdot$ Errors $\cdot$ Getting help $\cdot$ The Help Browser

\section{The Matbematica language}

\subsection{Expressions · 31}

Introduction - Internal forms of expressions - Atoms . The structure of expressions .

Exercises

2.2 Definitions 37

Defining variables and functions. Immediate vs. delayed assignments. The global rule base - Piecewise-defined functions . Functions with multiple definitions - Exercises

2.3 Predicates and Boolean operations · 45

Predicates $\cdot$ Relational and logical operators $\cdot$ Exercises

2.4 Attributes $\cdot 50$ 


\section{Lists}

3.1 Introduction $\cdot 53$

3.2 Creating and measuring lists . 55

List construction $\cdot$ Measuring lists $\cdot$ Exercises

3.3 Manipulating lists 60

Testing a list - Extracting elements $\cdot$ Rearranging lists $\cdot$ List component assignment . Exercises

3.4 Working with several lists $\cdot 69$

Exercises

3.5 Strings and characters $\cdot 70$

Exercises

\section{Functional programming}

\subsection{Introduction $\cdot 75$}

4.2 Functions for manipulating expressions $\cdot 77$

Map . Thread and MapThread . The Listable attribute - Apply Inner and Outer . Exercises

4.3 Iterating functions $\cdot 86$

Exercises

4.4 Programs as functions $\cdot 88$

User-defined functions $\cdot$ Building up programs $\cdot$ Exercises

4.5 Auxiliary functions 96

Compound functions - Localizing names: Module $\cdot$ Localizing values: Block .

Localizing constants: With $\cdot$ Exercises

4.6 Pure functions 102

Exercises

4.7 One-liners $\cdot 107$

Hamming distance $\cdot$ The Josephus problem $\cdot$ Pocket change $\cdot$ Exercises 


\section{$5 \quad$ Procedural programming}

5.1 Introduction $\cdot 115$

5.2 Loops and iteration 116

Newton's method $\cdot$ Do loops $\cdot$ While loops $\cdot$ NestWhile and NestWhileList $\cdot$ Exercises

5.3 Flow control • 131

Conditional functions . Multiclause definitions. Which and Switch P Piecewise .

Argument checking $\cdot$ Summary $\cdot$ Exercises

5.4 Examples · 142

Sieve of Eratosthenes $\cdot$ Classifying points $\cdot$ Exercises

\section{Rule-based programming}

\subsection{Introduction $\cdot 149$}

\subsection{Patterns 151}

Blanks $\cdot$ Sequence pattern matching $\cdot$ Example: Finding subsequences $\cdot$ Conditional pattern matching $\cdot$ Alternatives $\cdot$ String patterns $\cdot$ Exercises

6.3 Transformation rules 164

Introduction $\cdot$ Example: Counting coins $\cdot$ Example: Finding maxima $\cdot$ Exercises

6.4 Examples $\cdot 170$

Encoding text $\cdot$ Sorting a list $\cdot$ Exercises

\section{Recursion}

7.1 Fibonacci numbers $\cdot 177$

Exercises

7.2 List functions $\cdot 180$

Exercises

7.3 Thinking recursively: examples $\cdot 183$

Finding maxima $\cdot$ Subsets $\cdot$ Run-length encoding $\cdot$ Exercises

7.4 Recursion and symbolic computations 192

Exercises

7.5 Classical examples $\cdot 198$

Merge sort $\cdot$ Gaussian elimination - Trees $\cdot$ Huffman encoding $\cdot$ Exercises 
7.6 Dynamic programming $\cdot 216$

Exercises

7.7 Higher-order functions and recursion - 219

Exercises

\section{Numerics}

\subsection{Introduction $\cdot 221$}

\subsection{Numbers $\cdot 224$}

Types of numbers $\cdot$ Digits and number bases $\cdot$ Random numbers $\cdot$ Exercises

\subsection{Working with numbers $\cdot 234$}

Precision and accuracy $\cdot$ Representation of approximate numbers $\cdot$ Exact $v s$. approximate numbers $\cdot$ High precision vs. machine precision - Roundoff errors . Computing with different number types $\cdot$ Exercises

8.4 Working with arrays of numbers $\cdot 247$

Sparse arrays $\cdot$ Packed arrays $\cdot$ Exercises

\subsection{Numerical computations $\cdot 253$}

Working with precision and accuracy. Newton's method revisited $\cdot$ Gaussian elimination revisited $\cdot$ Exercises

\section{Graphics programming}

9.1 Structure of graphics $\cdot 269$

Primitives, directives, and options $\cdot$ Exercises

9.2 Graphics programming $\cdot 279$

Root plotting $\cdot$ Plotting data $\cdot$ Simple closed paths . Drawing trees $\cdot$ Exercises

9.3 Sound $\cdot 298$

The sound of mathematics $\cdot$ White noise, white music $\cdot$ Brownian music $\cdot$ Exercises

\section{Front end programming}

10.1 Introduction $\cdot 309$

10.2 The structure of cells and notebooks $\cdot 310$

Notebook expressions $\cdot$ Manipulating notebooks $\cdot$ Exercises 
10.3 Cell data types 319

TextData $\cdot$ BoxData $\cdot$ GraphicsData $\cdot$ Exercises

10.4 GridBoxes • 324

ShowTable $\cdot$ TriangleForm $\cdot$ Exercises

10.5 Buttons $\cdot 331$

Making buttons the easy way $\cdot$ The structure of buttons - ButtonStyle $\cdot$ ButtonFunction . Example: An evaluate button $\cdot$ Exercises

\section{Examples and applications}

\subsection{Manipulating data files $\cdot 341$}

Introduction - Getting the data into Mathematica $\cdot$ Examining the data file $\cdot$ Extracting and converting data $\cdot$ Visualizing the data $\cdot$ Exercises

11.2 Random walks $\cdot 351$

Introduction . The one-dimensional random walk . The two-dimensional random walk . Visualizing the random walk . The three-dimensional random walk Adding options and defaults · Error-trapping and messaging · Creating Help Browser documentation. Exercises

11.3 The Game of Life · 366

Exercises

11.4 Implementing languages $\cdot 374$

Introduction to PDL $\cdot$ Syntax $\cdot$ Parsing $\cdot$ Lexical analysis $\cdot$ Computing shapes

\section{Writing packages}

\subsection{Introduction $\cdot 395$}

12.2 Using packages $\cdot 396$

Loading packages $\cdot$ Finding out what is in a package $\cdot$ Avoiding name collisions

\subsection{Contexts · 401}

12.4 The elements of packages $\cdot 406$

12.5 Writing your own packages 408

The RandomWalks package $\cdot$ Exercises 
Appendix A How expressions are evaluated $\cdot 417$

Appendix B Debugging · 420

References $\cdot 427$

Solutions to exercises

2 The Matbematica language $\cdot 431$

3 Lists . 434

4 Functional programming 441

5 Procedural programming - 459

6 Rule-based programming $\cdot 468$

7 Recursion 480

8 Numerics 4992

9 Graphics programming 503

10 Front end programming $\cdot 522$

11 Examples and applications $\cdot 5291$

12 Writing packages $\cdot 537$

Index $\cdot 539$ 